\title{
Penalty Reduction in Industrial Power Consumption by Employing APFC Unit
}

\author{
G. Sravanthi ${ }^{1}$ \\ Assistant Professor, \\ Department of Electrical and Electronics Engineering, \\ Vignan Institute of Technology and Science, \\ Telangana.
}

\begin{abstract}
In the industrial division, number of motoring loads are running continuously. These motoring loads generate inductive load. Power consumption is increasing day by day at a very high rate. So, due to this inductive reactive power, the power factor gets reduced. The electricity company has specified certain limits, if the power factor gets below these specified limits penalty will be imposed to the industrial consumers. To overcome inductive reactance compensation is achieved by the use of power capacitors. APFC device reads the power factor from line voltage and current by determining the delay in arrival of the current signal with respect to voltage signal with high accuracy using internal timer. These values are calibrated as phase angle and corresponding power factor. Then the calculation for the requirement of compensation takes place in the motherboard and accordingly different capacitor banks are switched on.
\end{abstract}

Keywords- Power factor, inductive reactance, penalty, compensation, capacitors, capacitor banks.

\section{INTRODUCTION}

As most of the loads in modern electrical distribution systems are inductive, it is necessary to improve power factor. The low power factor of inductive loads robs system's capacity and may affect the voltage level. Power factor correction by the use of capacitors is widely in practice at all system voltages. As increase in utilities results in penalties, the electricity company charge customers for low power factor. The installation of power factor correction capacitors will improve the performance of the system and will save money.

Power factor theory: Power is classified into three types namely active power, reactive power and apparent power.

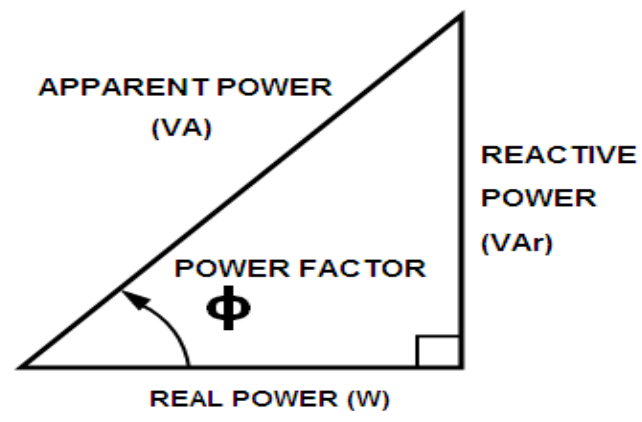

Figure 1: Power Triangle

\author{
P. Kovida Ramya ${ }^{2}$, K. Varalaxmi ${ }^{3}$, T. Dheeraj Kumar ${ }^{4}$ \\ U.G. Student, \\ Department of Electrical and Electronics Engineering, \\ Vignan Institute of Technology and Science, \\ Telangana.
}

Active Power: The actual amount of the power used or dissipated in a circuit is called active power or real power or true power. It's units are watts and is symbolized with the letter P.

Reactive Power: Reactive loads like inductors and capacitors dissipate zero power, but the fact that they drop voltage and draw current gives the perception that they dissipate power. This dissipated power is called reactive power. It's units are Volt-Amps-Reactive (VAR) and symbolized with the letter Q. It is the function of circuit's reactance $(\mathrm{X})$.

Apparent Power: The combination of the active and reactive power is called apparent power. It is the product of voltage and current of a circuit, without reference to phase angle. It's units are Volt-Amps (VA) and is symbolized with the letter S. Apparent power is a function of total impedance (Z) of a circuit.

Power factor: Power factor is defined as the ratio between the active power $(\mathrm{KW})$ to the total apparent power (KVA) consumed by an a.c. electrical equipment or a complete electrical installation. Power Factor is also defined as the cosine of the angle between current and voltage.

\section{Power Factor $(P F)=$ Active Power/Reactive Power}

It is a measure of how efficiently electrical power is converted into useful work output. Unity is the ideal power factor. If the power factor is less than one it means that extra power is required achieve the actual task at hand.

A poor power factor can be the result of either the significant phase difference between the voltage and current at the terminals of the load, or it can be because of the high harmonic content or distorted current waveform.

Causes of low power factor: Low power factor is due to the inductive loads. Inductive loads require the current to create a magnetic field that produces the desired work. This results in increase of reactive and apparent power and decreases the power factor, or efficiency of a system. An inductive load includes transformers, induction motors and induction generators, high intensity discharge lightening. These loads constitute distribution system. This increase in reactive power results in large angle between $\mathrm{KW}$ and KVA which further results in decrease of power factor.

Disadvantages of low power factor:

i. Large line losses: Line losses are proportional to the square of current. Therefore, larger the current, greater are the loine losses. 
ii. Greater conductor size and cost: At low power factor, current will be increased. To transmit this high current conductor size has to be increased.

iii. Effect on transformers: For decreased power factor, the KW capacity of the transformer is decreased and voltage is increased.

iv. Effect on switch gear and busbars: The crosssectional area of the bus bar, and the contact surface of the switchgears must be enlarged for the same power to be delivered at low power factors.

v. Poor voltage regulation and large voltage drop.

Advantages of power factor improvement:

i. The KW capacity of the prime movers is better utilized.

ii. This increases the kilowatt of the alternators.

iii. The efficiency of every plant is increased.

iv. The overall cost per unit is decreased.

v. $I^{2} R$ losses are reduced in transformers and distribution equipment.
Power factor correction: Capacitive power factor correction is applied to the circuits that include induction motors for reducing the inductive component of current and thereby reduces the losses in the supply. There should be no effect on the operation of the motor.

\section{PRINCIPLE}

Automatic Power Factor Correction (APFC) device is developed using AVR microcontroller. The voltage and current sample is converted into asquare wave by the use of zero cross detector. These samples are fed to microcontroller at INT0 and INT1 and calculates the difference between the arrival of the two waveforms which is the phase angle difference.

The difference is measured with high accuracy with the help of internal timer. This time values are calibrated as phase angle and corresponding power factor is also calculated. This value is displayed on the LCD module. Based on the requirement the capacitor banks are switched on in steps.

\section{METHODOLOGY}

\section{AUTOMATIC POWER FACTOR CORRECTOR USING} CAPACTTIVE LOAD BANK

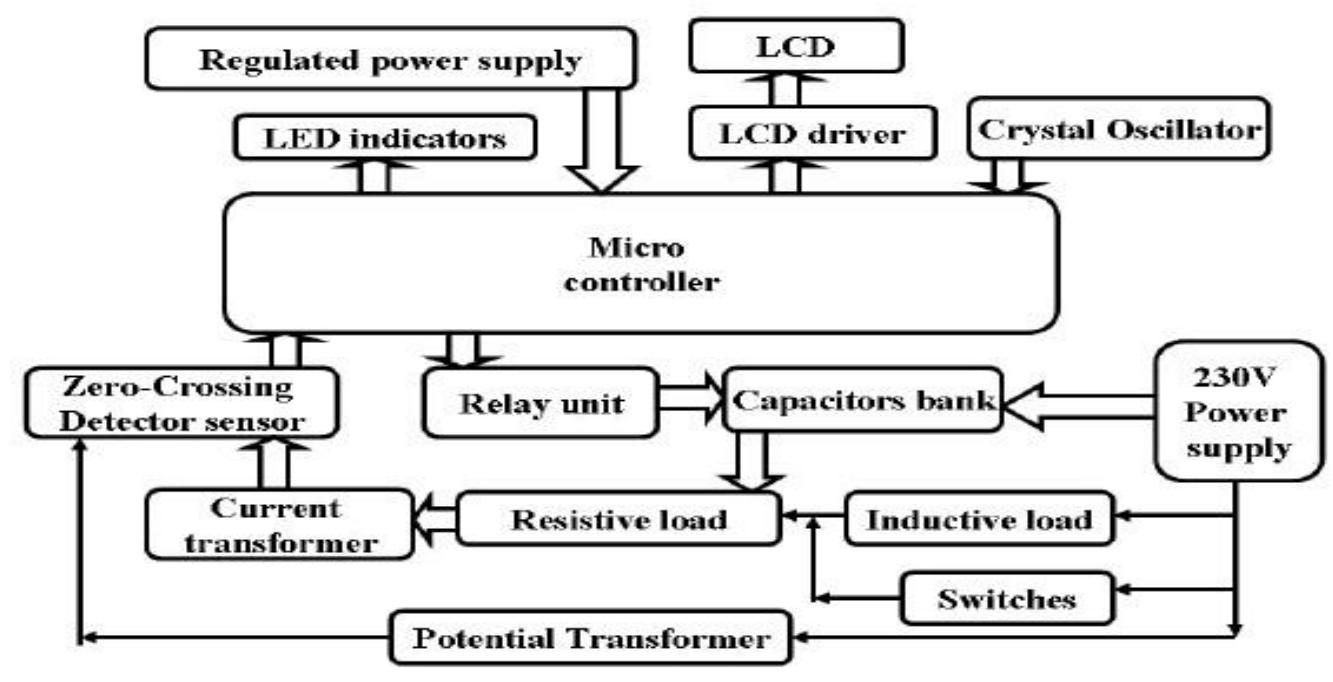

Figure 2: Automatic Power Factor Correction unit

Voltage and current estimation unit: Current transformer (CT) associated with line and the potential transformer (PT) is associated parallel with the supply line.

Comparator unit: It takes the values of voltage and current from PT and CT repectively and gives this information to the microcontroller.

Microcontroller (AVR): The ATmega328 is made by Atmel in the mega AVR family. It is utilized to perform various tasks and independent frameworks. Here, it calculates the phase delay between current and voltage and calibrates into phase angle and the corresponding power factor. Based on the calibration the capacitor banks are switched on.
Transformer: Transformers convert AC electricity from one voltage to another with little loss of power. Transformers work only with AC.

Relays: A relay is usually an electromechanical device that is actuated by an electrical current. The current flowing in one circuit causes the opening or closing of another circuit. Relays are like remote control switches and are used in many applications because of their relative simplicity, long life, and proven high reliability.

Choke coil: A coil is a series of loops. A coiled coil is a structure where the coil itself is in turn also looping. A coil is made up of materials, usually rigid, which can be fashioned into a spiral or helical shape. Flexible materials 
like wire, roe, hose, cable or paper can also be coiled into empty loops, or wound around central drum or spindle. This choke coil acts as the inductive load.

Capacitor Bank: The collection of capacitors of different values is known as capacitor bank. Series and parallel combination of different capacitors provide various ranges of capacitance required for the compensation of low power factor.

Calibration process: Few factors are necessary to be determined based on the components used in the voltage and current sensor circuits. These factors are considered in the calculation of accurate power. Voltage and current calibration constants are determined based on the stepdown and voltage divider ratios of PT and CT.

\section{KVAR Calculation:}

The calculated power parameters are used in the determination of the required KVAR. If the current power factor is $\cos \emptyset_{1}$ and targeted power factor is $\cos \emptyset_{2}$ then,

Required KVAR $=\mathrm{P}\left(\tan \varnothing_{1}-\tan \varnothing_{2}\right)$

Capacitance, $\mathrm{C}=\left(\mathrm{VAR} / 2 \pi \mathrm{fV}^{2}\right)$

Where, $\mathrm{P}$ is the Real power in $\mathrm{KW}$

$\mathrm{f}$ is the frequency

$\mathrm{V}$ is the voltage of the power system

Hardware requirement:

- Power supply

- LCD

- Relays

- Capacitors

- Resistors

- Transformer

- Voltage regulator

- Microcontroller

- Inductive load

- Voltage regulator

- Crystal oscillator

\section{Software requiement:}

- Embedded C

- Arduino software (IDE)

- Flash magic
IV. FLOWCHART

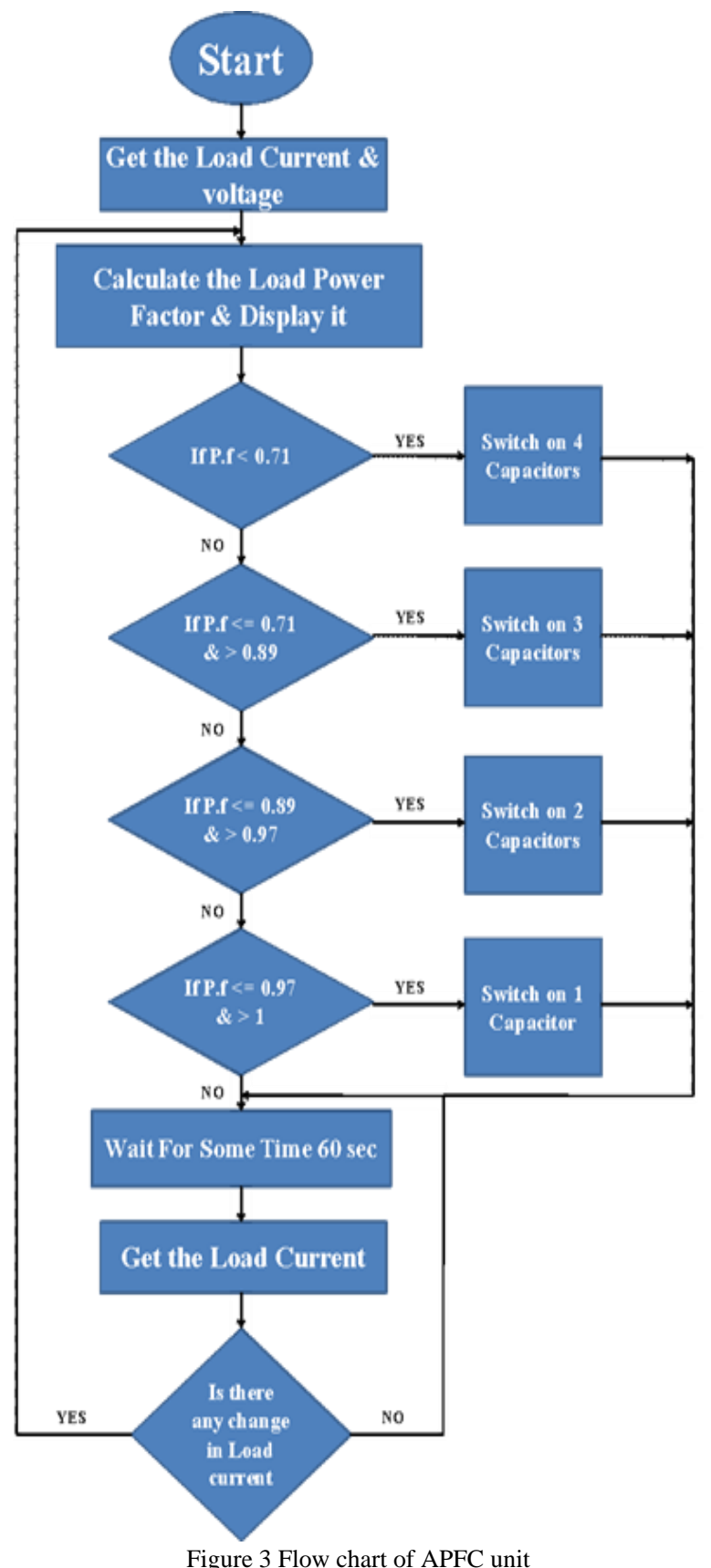

$\mathrm{V}$.

RESULT

1. Losses are reduced.

2. Efficiency is increased.

3. Reduction in electricity bills.

4. Better use of electrical machines and lines.

5. Power factor will be improved

6. Life span of the equipment is extended.

\begin{tabular}{|l|l|l|l|l|l|}
\hline \multicolumn{3}{|l|}{ Before Correction } & \multicolumn{3}{l|}{ After Correction } \\
\hline PF & I(A) & A.P.(VA) & PF & I(A) & $\begin{array}{l}\text { A.P. } \\
\text { (VA) }\end{array}$ \\
\hline 0.85 & 0.77 & 171 & 0.96 & 0.70 & 156 \\
\hline 0.72 & 1.02 & 229 & 0.96 & 0.79 & 177 \\
\hline 0.60 & 1.22 & 271 & 0.96 & 0.80 & 178 \\
\hline 0.50 & 1.35 & 301 & 0.95 & 0.76 & 172 \\
\hline
\end{tabular}




\section{CONCLUSION}

It can be concluded that power factor correction procedures can be connected to industries, power system and furthermore households to make them stable and because of that the framework winds up stable efficiency of the system and in addition mechanical assembly increases.

It dispenses the technique used to overcome the penalty imposed and power loss due to to low power factor indefinite with same residential and small industrial unit. The static capcitor is used in industries to improve the power factor in industry and distribution lines. Thus, it not only imprves power factor but also increases line capacityadn efficiency.

\section{REFERENCES}

[1] An Introduction to Embedded Systems Florian Lechner, Daniel Walter csad5478@uibk.ac.at, csae8958@uibk.ac.at November 8, 2006

[2] What is Reactive Power? Peter W. Sauer Department of Electrical and Computer Engineering University of Illinois at UrbanaChampaign September 16, 2003.
[3] Hubbi Walid, and Takashi Hiyama, "Placement of static compensator to minimize power system losses ", Electric power system research, Vol.47, issue 2, 1998.

[4] Tiwari Anant kumar., Sharma, Durga., and sharama, Vijay kumar., "Automatic power factor correction using capacitive bank", Dr. C.V.Raman Institute of science and technology,Vol.4, pp.394-395, 2014.

[5] Oscar Garcia, Member, IEEE, Joes A. obos, Member, IEEE Robert Prieto, Member, IEEE, Pedro Alou,Members of IEEE, Javier Uceda,Senior Member of

[6] IEEE "Single Phase Power Factor Correction :A Survey",IEEE TRANSACTIONS ON POWER ELECTRONICS, Volume 18 No3, IssuedMay 2003.

[7] IEEE TRANSACTIONS ON INDUSTRIAL ELECTRONIC VOL NO 3 FEBRUARY $1990 \quad 77 \mathrm{~A}$ Microprocessor-Based Adaptive Power Factor Corrector for Nonlinear Loads H. M. ELBOLOK,M. E. MASOUD, AND M. M.MAHMOU

[8] Jain Sandesh, Thakur Shivendra Singh and Phulambrikar S.P., "Improve Power Factor And Reduce the Harmonic Distortion of the System", International Journal of Advanced Research in Computer Science and Software Engineeriong. Volume 1(5), issued November 2012.

[9] Electric Power Systems by S.L.Uppal.

[10] Utilization of Electric Power and Electric Traction by J.B.Gupta. 